\title{
PINNED EQUIVALENCE RELATIONS
}

\author{
JINDŘICH ZAPLETAL
}

\begin{abstract}
Answering a question of Kechris, I show that the equality of countable sets
\end{abstract} of reals is not the smallest unpinned equivalence relation.

\section{Introduction}

The theory of Borel reducibility of equivalence relations on Polish spaces has grown considerably in the previous two decades $[3,1]$. It all revolves around the following central definition:

Definition 1.1. Let $E, F$ be equivalence relations on respective Polish spaces $X, Y$. The equivalence $E$ is Borel reducible to $F$ if there is a Borel function $g: X \rightarrow Y$ such that $\forall x_{0}, x_{1} \in X x_{0} E x_{1} \leftrightarrow g\left(x_{0}\right) F g\left(x_{1}\right)$.

The Borel reducibility is a quasiordering comparing the equivalence relations in the sense of their intuitive complexity. Mathematicians started building a map of the commonly used Borel or analytic equivalence relations under this quasiordering. In order for this program to succeed, it needs tools for proving that a given Borel equivalence relation is not reducible to another one. One such fairly useful tool is the notion of pinned/unpinned equivalence relation.

Definition 1.2. [3, Chapter 17] A Borel equivalence relation $E$ on a Polish space $X$ is unpinned if there is a forcing $P$ and a $P$-name $\dot{x}$ for an element of $X$ such that $P \times P$ forces the left and right evaluations of $\dot{x}$ to be $E$ equivalent, and $P$ forces $\dot{x}$ to be $E$-inequivalent to any ground model point of the space $X$. The equivalence is pinned if it is not unpinned.

It is not difficult to see that if a Borel equivalence relation is pinned, then so are all of the equivalence relations Borel reducible to it. This leads for example to a short proof that $F_{2}$, the equivalence on infinite sequences of reals connecting two such sequences if their ranges are the same, is not reducible to $E_{l_{\infty}}$, the equivalence on infinite set of reals connecting two of them if their difference is bounded. The point is that $E_{l_{\infty}}$ is pinned due to the complexity of its definition [3, Theorem 17.1.3], while $F_{2}$ is unpinned: the forcing witnessing the requisite property of $F_{2}$ is the collapse of $2^{\omega}$ to countable size, with $\dot{x}$ a name for an enumeration of the set of ground model points

Received by the editors December 17, 2010.

2000 AMS subject classification. 03E40, 03E15. 
of $2^{\omega}$. Both of these equivalence relations occupy strategic positions in the Borel reducibility map, and occur naturally in many places. For example, the Halmos-von Neumann theorem shows that the conjugacy of ergodic measure preserving transformations with discrete spectrum is bireducible with $F_{2}$; the Lipschitz isomorphism of compact metric spaces is bireducible with $E_{l_{\infty}}[7]$.

One difficulty with the notion of an unpinned equivalence relation is its complex definition. This may lead to the undesirable effect that for some Borel equivalence relation, the status of pinned/unpinned may not be invariant under forcing. Kechris asked whether perhaps this is an irrelevant issue and the unpinned property is simply equivalent to $F_{2}$ being reducible to a given Borel equivalence relation:

Question 1.3. [3, Question 17.6.1] Is $F_{2}$ reducible to every unpinned Borel equivalence relation?

In this brief note, I will show that the answer is negative.

Theorem 1.4. There is an unpinned Borel equivalence relation strictly below $F_{2}$ in the Borel reducibility sense.

In fact, it turns out that there is a whole small universe of heretofore unknown unpinned equivalence relations strictly below $F_{2}$, with a complex structure related to small uncountable cardinals.

The notation in this paper follows the set theoretic standards of [2].

\section{Proof}

Motivated by model theoretic concerns, Shelah [8] showed that there are Borel sets in the plane with unexpectedly complex behavior:

Fact 2.1. For every countable ordinal $\alpha \in \omega_{1}$ there is a c.c.c. extension in which continuum is large and there is an $F_{\sigma}$ set $B_{\alpha} \subset 2^{\omega} \times 2^{\omega}$ which has a clique of size $\aleph_{\alpha}$ but no larger clique.

Here, a clique for a set $B \subset 2^{\omega} \times 2^{\omega}$ is simply a set $C \subset 2^{\omega}$ such that $C \times C \subset B$. Later on, in a joint work with Kubis [5] the offending $F_{\sigma}$ sets were constructed in ZFC in the ground model, so that the c.c.c. extension is only used to increase the size of the continuum in a suitable way and not to obtain the Borel codes of these sets. A particularly simple example was found by Kubis and Vejnar [6]:

Fact 2.2. There are continuous functions $f_{n}: n \in \omega$ from $2^{\omega}$ to $2^{\omega}$, such that the union $B$ of their graphs and graphs of their inverses has an uncountable clique.

The proof is so simple and instructive that it should be reproduced here. Let $\omega=\bigcup_{n} a_{n}$ be a partition of $\omega$ into infinite sets, with $\pi_{n}: \omega \rightarrow a_{n}$ an increasing enumeration of each, and let $f_{n}(x)=x \circ \pi_{n}$. Let $B$ be the union of graphs of $f_{n}$ 's and their inverses and the diagonal. Now argue that every maximal clique of $B$ must be in fact uncountable. If $\left\{x_{m}: m \in \omega\right\}$ is a clique, it is easy to find a point $y \in 2^{\omega}$ such that $\forall m x_{m}=f_{m+1}(y)$ and $y\left\lceil a_{0} \notin\left\{x_{m}\left\lceil a_{0}: m \in \omega\right\}\right.\right.$. Then, $\left\{y, x_{m}: m \in \omega\right\}$ is a clique extending the original countable clique!

It is clear that a set $B$ as described in this Fact cannot have a clique of size $\aleph_{2}$. If $C=\left\{x_{\alpha}: \alpha \in \omega_{2}\right\}$ was such a clique then on cardinality grounds there would be 
an ordinal $\beta \in \omega_{2}$ such that for no $\gamma \in \omega_{1}$, no $\delta \in \omega_{2} \backslash \beta$ and no $n \in \omega$ it is the case that $f_{n}\left(x_{\gamma}\right)=\delta$. On cardinality ground again, there would be an ordinal $\gamma \in \omega_{1}$ such that for no $n \in \omega$ and no $\delta \in \omega_{1} \backslash \gamma$ it is the case that $f_{n}\left(x_{\beta}\right)=x_{\delta}$. Then clearly the point $\left\langle x_{\gamma}, x_{\beta}\right\rangle$ cannot belong to the set $B$, contradicting the assumption that $C$ was a clique.

Fix a set $B \subset 2^{\omega} \times 2^{\omega}$ as provided by the fact. Let $Y$ be the Borel set of those sequences $y \in\left(2^{\omega}\right)^{\omega}$ whose range is a clique in $B$, and let $E=F_{2} \uparrow Y$. It is immediate that $E \leq F_{2}$; the identity is the reducing function. I will show that $E$ is unpinned and $F_{2}$ does not reduce to $E$; this will complete the proof of the theorem.

First of all, the equivalence $E$ is unpinned. Let $C \subset 2^{\omega}$ be an uncountable clique of $B$, let $P$ be a forcing enumerating the set $C$ in ordertype $\omega$, and let $\dot{y}$ be the $P$-name for the generic enumeration. It is immediate that $\dot{y}$ witnesses the requisite property of the equivalence $E$.

To show that $F_{2}$ is not reducible to $E$, suppose that $f$ is such a Borel reduction and work towards a contradiction. Move to a generic extension $V[G]$ where the continuum hypothesis fails. Note that $f$ remains a reduction in $V[G]$ by Shoenfield's absoluteness, and the set $B$ still has no clique of size $\aleph_{2}$. Consider the forcing $P$ collapsing the size of the continuum to $\aleph_{0}$ and let $\dot{x}$ be a name for the generic enumeration of the ground model elements of $2^{\omega}$.

Claim 2.3. $P \Vdash \operatorname{rng}(f(\dot{x})) \in V$.

Proof. It must be the case that $P \Vdash \operatorname{rng}(f(\dot{x})) \subset V$. If some condition forced a new element into the set, one could pass to a forcing extension with mutually generic filters $H_{0}, H_{1} \subset P$ containing that condition. Clearly, $\left(\dot{x} / H_{0}\right) F_{2}\left(\dot{x} / H_{1}\right)$, but the ranges of $f\left(\dot{x} / H_{0}\right)$ and $f\left(\dot{x} / H_{1}\right)$ are not equal by a mutual genericity argument. Thus $f$ would not be a reduction in that extension.

It also must be the case that for every ground model element $y \in Y$, the largest condition in $P$ must decide the statement $\check{y} \in \operatorname{rng}(f(\dot{x}))$. If $p, q \in P$ decided this statement in two different ways, then one could pass into a forcing extension with $V[G]$-generic filters with $p \in H_{0}, q \in H_{1}$. But then, $\left(\dot{x} / H_{0}\right) F_{2}\left(\dot{x} / H_{1}\right)$ while $y \in$ $\operatorname{rng}\left(f\left(\dot{x} / H_{0}\right)\right) \Delta \operatorname{rng}\left(f\left(\dot{x} / H_{1}\right)\right)$ and $f$ is not a reduction in this extension.

Consequently, $P \Vdash \operatorname{rng}(f(\dot{x}))=\{y: 1 \Vdash \check{y} \in \operatorname{rng}(\dot{x})\} \in V$.

Let $C \subset 2^{\omega}$ be the set forced to be the range of $\dot{f}(\dot{x})$. Plainly, $C$ is a clique in $B$, and therefore its size is at most $\aleph_{1}<\mathfrak{c}$. Thus there are two elementary submodels $M_{0}, M_{1}$ of a large enough structure which contain $C$ as an element and a subset such that $M_{0}, M_{1}$ do not contain the same reals. Pass into a forcing extension $V[G][H]$ in which there are filters $H_{0} \subset M_{0} \cap P$ and $H_{1} \cap M_{1} \cap P$ meeting all the dense sets in the respective models. By the forcing theorem applied in the models, $M_{0}\left[H_{0}\right] \models \operatorname{rng}\left(f\left(\dot{x} / H_{0}\right)\right)=C$ and $M_{1}\left[H_{1}\right] \models \operatorname{rng}\left(f\left(\dot{x} / H_{1}\right)\right)=C$, and by Borel absoluteness between the models $M_{0}\left[H_{0}\right], M_{1}\left[H_{1}\right]$ and the extension, it is the case that $\operatorname{rng}\left(f\left(\dot{x} / H_{0}\right)\right)=C=\operatorname{rng}\left(f\left(\dot{x} / H_{1}\right)\right)$. However, the sequences $\dot{x} / H_{0}, \dot{x} / H_{1}$ are $F_{2}$ inequivalent, since the models $M_{0}, M_{1}$ did not contain the same reals. Thus $f$ is not a reduction in the generic extension $V[G][H]$, a contradiction. The theorem follows! 


\section{Concluding remarks}

The result opens more questions than it resolves. Let $B \subset 2^{\omega} \times 2^{\omega}$ be a Borel set, and $E_{B}$ is the equivalence relation $F_{2}$ restricted to the Borel set of all sequences $\vec{x} \in\left(2^{\omega}\right)^{\omega}$ enumerating cliques of $B$. What are the reducibility connections between various equivalences of the form $E_{B}$ and other Borel equivalence relations?

Claim 3.1. The equivalence relation $E_{B}$ is pinned if and only if every clique of $B$ is countable.

Proof. Obviously, every uncountable clique yields an unpinning forcing which just enumerates the clique generically in type $\omega$. On the other hand, if every clique is countable, $P$ is a forcing and $\tau$ is a $P$-name for an enumeration of a clique such that $P \times P \Vdash \operatorname{rng}\left(\tau_{\text {left }}\right)=\operatorname{rng}\left(\tau_{\text {right }}\right)$, then similarly to Claim 2.3 there must be a clique $C$ such that $P \Vdash \operatorname{rng}(\tau)=\breve{C}$, and since $C$ is countable, $P \Vdash \tau$ is equivalent to any enumeration of the clique $C$.

Having an uncountable clique is a property of a Borel set $B$ which is invariant under forcing. It is equivalent to an existence of a model of a certain sentence in the language $L_{\omega_{1} \omega}(Q)$, where $Q$ is the quantifier "there exist uncountably many". However, Keisler [4] showed that the relevant infinitary logic is complete, and so the existence of a model is equivalent to the consistency of the sentence. The consistency means nonexistence of a proof of contradiction, the proofs are wellordered countable sequences of countable formulas, and so the consistency of such a theory is equivalent to a $\Pi_{2}^{1}$ sentence, and therefore absolute between transitive models of set theory.

Claim 3.2. $F_{2}$ is reducible to the equivalence relation $E_{B}$ if and only if $B$ has a perfect clique.

Proof. On one hand, if $C \subset 2^{\omega}$ is a perfect clique, then choose a Borel injection $f: 2^{\omega} \rightarrow C$ and naturally extend it to a function $f:\left(2^{\omega}\right)^{\omega} \rightarrow\left(2^{\omega}\right)^{\omega}$ by $f\left(x_{n}: n \in\right.$ $\omega)=\left(f\left(x_{n}\right): n \in \omega\right)$. The function $f$ is a Borel reduction of $F_{2}$ to $E_{B}$.

On the other hand, suppose that $B$ has no perfect clique and $f$ is a Borel reduction of $F_{2}$ to $E_{B}$. I will reach a contradiction by passing into a large Cohen extension $V[G]$ and showing that in it, $B$ has no clique of size $\boldsymbol{c}$. The function $f$ should still be a reduction in the model $V[G]$ by analytic absoluteness, but then a contradiction is reached just as in the previous section.

The proof that in a large Cohen extension the set $B$ cannot have a clique of size continuum can be found in several places in the literature, including Shelah's [8]. Work in $V$ and let $\kappa$ be a regular cardinal larger than the continuum such that $\kappa^{\omega}=\kappa$. The model $V[G]$ is the extension of $V$ with forcing $Q$ adding $\kappa$ many Cohen reals with finite support. To verify the requisite feature, suppose for contradiction that the poset $Q$ forces that $\left\langle\dot{z}_{\alpha}: \alpha \in \kappa\right\rangle$ is a clique in the set $B$. For every ordinal $\alpha \in \kappa$, let $M_{\alpha}$ be a countable elementary submodel of a large structure containing $\alpha$. Note that the c.c.c. of $Q$ implies that $\dot{z}_{\alpha} \cap M_{\alpha}=\dot{z}_{\alpha}$ for every ordinal $\alpha$. Use the cardinal arithmetic assumption to find a cofinal set $a \subset \kappa$ such that the models $M_{\alpha}: \alpha \in a$ form a $\Delta$-system with root $r$. Thinning out the set $a$ further if necessary I may assume that the structures $\left\langle M_{\alpha}, \dot{z}_{\alpha}, r, \dot{u}_{\alpha}\right\rangle: \alpha \in a$ are pairwise isomorphic, with the same transitive collapse $\bar{M}, \bar{z}, \bar{r}, \bar{u}$. The simple form of the forcing $Q$ implies that $Q \cap r$ is a regular subposet of $Q \cap \bar{M}$ which is in turn regular in $Q$ and so there is 
a $Q \cap r$ name $\dot{u}_{\alpha}$ for the remainder of the name $z_{\alpha}$. Now, for every pair of ordinals $\alpha \neq \beta \in a, Q$ forces that the filters $\dot{G} \cap M_{\alpha} \backslash r$ and $\dot{G} \cap M_{\beta} \backslash r$ are mutually generic over $V[\dot{G} \cap r]$, and the evaluations of the names $\dot{u}_{\alpha}, \dot{u}_{\beta}$ according to these filters provide a pair of points in the set $B$. It follows that in the model $V[G \cap r]$, it is the case that the product of two copies of the poset $Q \cap \bar{M} \backslash \bar{r}$ force the two evaluations of the name $\bar{u}$ to form a point in the set $B$; moreover, the evaluations must be distinct by a mutual genericity argument. Back to the ground model $V$. choose a countable elementary submodel $N$ of a large structure containing the transitive collapses. It is easy to find an $N$-generic filter $g \subset \bar{Q} \cap \bar{r}$ and a perfect set $D$ of pairwise $\bar{N}[g]$-generic filters on $\bar{Q} \backslash \bar{r}$, and any perfect subset of the uncountable analytic set $\{\bar{z} / g \cup h: h \in D\}$ is a perfect clique of $B$ by the forcing theorem applied to $N$ and an absoluteness argument. A contradiction!

Since all Borel countable equivalence relations are reducible to $F_{2}$, it is natural to wonder whether they are also reducible to the relations $E_{B}$. Regarding this question, I will just show that it is not difficult to amend the set $B$ from the previous section so that all countable equivalence relations reduce to $E_{B}$. To do that, let $E$ be a universal countable Borel equivalence relation on $2^{\omega}$, fix an action of a countable group $G$ whose orbit equivalence $E$ is, and enrich the countable set $\left\{f_{n}: n \in \omega\right\}$ of functions generating the set $B$ by the functions $x \mapsto g \cdot x$ for all $g \in G$. The resulting set $B$ is still strictly below $F_{2}$ by the same proof, and the function $x \mapsto\langle g \cdot x: g \in G\rangle$ reduces $E$ to $E_{B}$.

The structure of the equivalence relations of the form $E_{B}$ under the Borel reducibility quasiorder seems to be very complex. I will conclude the paper with noting that if a set $B$ has a clique of certain cardinality, then it is not reducible to any equivalence reduction $E_{C}$ where $C$ has no clique of that cardinality, as the proof in the previous section immediately shows. Thus, the results of Shelah and Kubis $[8,5]$ yield uncountably many unpinned Borel equivalence relations below $F_{2}$, pairwise not bireducible to each other.

\section{Acknowledgements}

The author's research has been partially supported by NSF grant DMS 0801114 and Institutional Research Plan No. AV0Z10190503 and grant IAA100190902 of GA AV ČR. The author also acknowledges the support of grants MEB060909 and MEB051006 of MŠMT ČR.

\section{References}

[1] Su Gao. Invariant descriptive set theory. CRC Press, Boca Raton, 2009.

[2] Thomas Jech. Set Theory. Academic Press, San Diego, 1978.

[3] Vladimir Kanovei. Borel equivalence relations. University Lecture Series 44. American Mathematical Society, Providence, RI, 2008.

[4] Jerome Keisler. Logic with quantifier "there exist uncountably many". Annals of Mathematical Logic, 1:1-93, 1970. 
[5] Wieslaw Kubis and Saharon Shelah. Analytic colorings. Annals of Pure and Applied Logic, 121:145-161, 2003. math.LO/0212026.

[6] Wieslaw Kubis and Benjamin Vejnar. Covering an uncountable square by countably many functions. ArXiv 0710.1402, 2007.

[7] Christian Rosendal. Cofinal families of Borel equivalence relations and quasiorders. J. Symbolic Logic, 70:1325-1340, 2005.

[8] Saharon Shelah. Borel sets with large squares. Fundamenta Mathematicae, 159:1-50, 1999. math.LO/9802134.

Institute of Mathematics, Academy of Sciences, Czech Republic, Žitná 25, CZ - 115 67 Praha 1, Czech Republic and Department of Mathematics, University of Florida, Gainesville FL 32611-8105, USA

E-mail address: zapletal@math.cas.cz 